J. Dairy Sci. 97:1999-2008

http://dx.doi.org/10.3168/jds.2013-7470

(C) American Dairy Science Association ${ }^{\circledR}, 2014$.

\title{
Quantitative profiling of bacteriocins present in dairy-free probiotic preparations of Lactobacillus acidophilus by nanoliquid chromatography-tandem mass spectrometry
}

\author{
Renu Nandakumar*1 and Kesh Talapatra† \\ ${ }^{*}$ Proteomics and Metabolomics Core Facility, Redox Biology Center, Department of Biochemistry, University of Nebraska-Lincoln, Lincoln 68588 \\ †Natren Inc., 2382 Townsgate Rd, Westlake Village, CA 91361
}

\begin{abstract}
Bacteriocins are a heterogeneous group of ribosomally synthesized peptides or proteins with antimicrobial activity, produced predominantly by lactic acid bacteria, with potential applications as biopreservatives and probiotics. We describe here a novel strategy based on a bottom-up, shotgun proteomic approach using nanoliquid chromatography-tandem mass spectrometry (nanoLC-MS/MS) with multiple fragmentation techniques for the quantitative profiling of bacteriocins present in the probiotic preparations of Lactobacillus acidophilus. A direct LC-MS/MS analysis with alternate collision-induced dissociation, high-energy collision dissociation, and electron-transfer dissociation fragmentation following a filter-assisted size-exclusion sample prefractionation has resulted in the identification of peptides belonging to 37 bacteriocins or related proteins. Peptides from lactacin F, helveticin J, lysin, avicin A, acidocin M, curvaticin FS47, and carocin D were predominant. The process of freeze drying under vacuum was observed to affect both the diversity and abundance of bacteriocins. Data acquisition using alternating complementary peptide fragmentation modes, especially electron-transfer dissociation, has significantly enhanced the peptide sequence coverage and number of bacteriocin peptides identified. Multienzyme proteolytic digestion was observed to increase the sample complexity and dynamic range, lowering the chances of detection of low-abundant bacteriocin peptides by LC-MS/MS. An analytical platform integrating size exclusion prefractionation, nanoLC-MS/MS analysis with multiple fragmentation techniques, and data-dependent decision tree-driven bioinformatic data analysis is novel in bacteriocin research and suitable for the comprehensive bioanalysis of diverse, low-abundant bacteriocins in complex samples.
\end{abstract}

Received September 9, 2013.

Accepted December 28, 2013.

${ }^{1}$ Corresponding author: rnandakumar2@unl.edu
Key words: bacteriocin, Lactobacillus acidophilus, nanoliquid chromatography-tandem mass spectrometry, electron-transfer dissociation

\section{INTRODUCTION}

Bacteriocins constitute a heterogeneous group of ribosomally synthesized antibacterial peptides or proteins displaying antagonistic activity, barring few exceptions, against species closely related to producer strains that have a specific immunity mechanism (Klaenhammer, 1993; Sawa et al., 2013). They have drawn increased attention in recent years due to their potential applications in the food and pharmaceutical industry as biopreservatives and therapeutic antibiotics (Riley and Wertz, 2002; Chen and Hoover, 2003; Urbizu et al., 2013).

Bacteriocins differ widely based on their structure, mode of action, antimicrobial potency, immunity mechanisms, and target cell receptors (Gillor et al., 2008). They are classified mainly into 3 groups with respect to their primary structure, molecular mass, and heat stability: lantibiotics containing posttranslationally modified amino acid residues, lanthionine (class I); small $(<10 \mathrm{kDa})$ heat-stable non-lantibiotics (class II), further divided into subgroups pediocin-like and anti-Listeria bacteriocins (subclass IIa), the 2-peptide bacteriocins (subclass IIb), and the sec-dependent bacteriocins (subclass IIc); and the large $(>30 \mathrm{kDa})$ heatlabile non-lantibiotics (class III) (Cintas et al., 2001; Cotter et al., 2005; Deraz et al., 2005). Most bacteriocins characterized to date belong to class II and act on sensitive cells by destabilization and permeabilization of the cytoplasmic membrane through the formation of transitory poration complexes or ionic channels that cause the reduction or dissipation of the proton motive force (Abee, 1995). Bacteriocins and their mode of action, antimicrobial spectrum, and biosynthesis were extensively reviewed by Jack et al. (1995) and Cintas et al. (2001).

Lactic acid bacteria (LAB) with the generally recognized as safe (GRAS) status play an important role 
in food industry due to their beneficial effects on nutritional, organoleptic, and shelf-life characteristics of food products and as components of many probiotic supplements. The probiotic effect of LAB is attributed to their ability to strengthen the intestinal barrier, to modulate the host immune system, and to produce antimicrobial substances (Corr et al., 2009). Bacteriocin production may positively affect the ability of a probiotic strain to compete within the complex gut microbiome, as it may facilitate the modulation of the gut microbiota composition and the host immune system (Dobson et al., 2012). Lactobacillus acidophilus is a strain widely used in functional foods and dietary supplements in a global probiotics market expected to be worth $\$ 32.6$ billion by 2014 (MarketsandMarkets, 2009). The majority of bacteriocins produced by L. acidophilus are heat-stable, low-molecular-mass, non-lantibiotic peptides belonging to class II (Zamfir et al., 1999).

Recently, bacteriocin production has been suggested as an important probiotic trait, making it essential to incorporate a screening step for bacteriocin production during the selection phase of a novel probiotic or to reassess the health benefits of an existing probiotic strain. Bioanalysis and characterization of low-abundant, small-molecular-weight class of bacteriocins is an analytical challenge and different formats of functional assays and immunoassays are in practice (Nandakumar et al., 2000; Mugochi et al., 2001). The technical challenges will be amplified when it comes to analysis of probiotic products due to the interference from media components and other proteinaceous materials. In the past, several studies have used mass spectrometry-based methods such as matrix-assisted laser desorption/ionization time-of-flight mass spectrometry (MALDI-TOF-MS; Rose et al., 1999; Hindré et al., 2003) and, lately, electrospray ionization-liquid chromatography-mass spectrometry (ESI-LC-MS; Zendo et al., 2008; Malini and Janakiraman, 2012) for the detection and molecular mass determination of bacteriocins. The bottom-up, quantitative proteomics approach using liquid chromatography (LC) for peptide separation, followed by tandem mass spectrometry (MS/MS) and bioinformatics analysis will allow more sensitive detection, sequence information, and relative quantitation of low-abundant, small-molecular-weight proteins or peptides in a complex sample (Aebersold and Mann, 2003; Nesvizhskii, 2007). Recently, electron-transfer dissociation (ETD) was developed as an alternative peptide-fragmentation method and was shown to generate more complete series of ions and, thus, more extensive sequence information, while preserving labile posttranslational modifications (Good et al., 2007). In this paper, we detail the firsttime application of nanoliquid chromatography-MS/
MS (nanoLC-MS/MS) with alternating multiple peptide-fragmentation methods for the quantitative profiling of bacteriocins present in probiotic preparations of L. acidophilus.

\section{MATERIALS AND METHODS}

\section{Samples}

Four different probiotic samples obtained from Natren Inc. (Westlake Village, CA) were analyzed for the presence of bacteriocins. The medium (Natren 1, N1) used for the cultivation of L. acidophilus NAS with a nutritionally balanced formula containing protein from garbanzo beans, carbohydrates, vitamins, and minerals was treated as the control. Other samples included (a) culture medium after bacterial growth (N2), (b) bacterial culture medium after freeze drying under vacuum (N3), and (c) a freeze-dried probiotic preparation of the L. acidophilus NAS strain with a different product formulation obtained from a commercial source (NATSCO). The details of this manufacturer, culture media composition, and freeze-drying process parameters used for the preparation of NATSCO are not disclosed to preserve the company's confidentiality. The latter probiotic preparation was primarily included to evaluate the effect of bacterial growth and process parameters on the bacteriocin profile as well as the sensitivity and robustness of the developed LC-MS/ MS workflow to analyze probiotic samples of varying complexity.

\section{Freeze Drying Under Vacuum}

Following bacterial cultivation, the Natren formulations were freeze-dried using a commercial freeze dryer (BOC Edwards Pharmaceutical Systems, Tonawanda, NY). The complete freeze-drying process included the following stages: (a) pretreatment, (b) freezing, (c) primary drying, and (d) secondary drying. Pretreatment was primarily included to increase and (or) improve stability/processing by decreasing the high-vapor-pressure solvent or increasing the surface area by adding a cryoprotectant. The material was cooled to ensure that sublimation rather than melting occurred. The freezing was done quickly between -50 and $-80^{\circ} \mathrm{C}$ to avoid the formation of large ice crystals. The primary drying was conducted by lowering the pressure to 10 to $20 \mathrm{kPa}$ and providing enough heat for water to sublime. This phase of the process was slow, taking 1 to $3 \mathrm{~d}$. The application of vacuum sped up the sublimation. The condenser temperatures were typically below $-50^{\circ} \mathrm{C}$. The secondary drying phase was used to remove the unfrozen water molecules, as the ice was removed in 
the primary drying. The temperature was increased, ranging from 0 to $30^{\circ} \mathrm{C}$ for 2 to $12 \mathrm{~h}$. Upon completion of the freeze-drying process, the vacuum was broken by using nitrogen. The freeze-drying process time ranged from 26 to $44 \mathrm{~h}$. The moisture content of the freezedried product was approximately 1 to $4 \%$.

\section{Sample Preparation}

Because bacteriocins are a very heterogeneous group comprising a dynamic range of peptides/proteins of molecular weights ranging from $<5$ to $10 \mathrm{kDa}$ up to $90 \mathrm{kDa}$, a filter-assisted size-exclusion protein fractionation and acetone precipitation for selective enrichment were included. Multi-enzyme proteolytic digestion was carried out for better sequence coverage of larger peptides/proteins. Two workflows were devised for the LCMS/MS analysis: workflow 1 (direct LC-MS/MS) for the enhanced detection of proteins/peptides of $\leq 3 \mathrm{kDa}$ in size and workflow 2 for the identification of larger bacteriocins (shotgun LC-MS/MS).

\section{Direct LC-MS/MS}

Twenty milliliters each of samples N1 and N2 was thawed to room temperature and centrifuged at 16,000 $\times g$ for $20 \mathrm{~min}$ at $4^{\circ} \mathrm{C}$ to remove microbial cells and other particles. One gram each of the powder formulations of samples N3 and NATSCO was resuspended in HPLC-grade water by vortexing and centrifuged at $16,000 \times g$ for $20 \mathrm{~min}$ at $4^{\circ} \mathrm{C}$ to remove microbial cells. Supernatants from the samples were recentrifuged at $16,000 \times g$ for 15 min to improve the clarity of the solutions and were used for further size-exclusion separation steps. Protein concentrations in the samples were measured using a Pierce BCA protein assay kit (Thermo Scientific, Rockford, IL). Initially, sample supernatants containing $250 \mu \mathrm{g}$ of protein were filtered through a 10-kDa cutoff Amicon Ultra centrifugal filter (Millipore Corp., Billerica, MA) to remove high-molecular-weight proteins and to reduce sample complexity. The collected filtrates containing $\leq 10-\mathrm{kDa}$ proteins were passed again through a 3 -kDa cutoff filter membrane (Amicon; Millipore Corp.) to further fractionate and concentrate the peptides with a molecular weight $\leq 3 \mathrm{kDa}$. The filtrate containing peptides $\leq 3 \mathrm{kDa}$ was dried in a speed vacuum concentrator, resuspended in $0.1 \%$ formic acid, and used directly for LC-MS/MS analysis. In addition, the filter retentate containing $\geq 3$ - $\mathrm{kDa}$ proteins was also dried, resuspended in $100 \mathrm{~m} M$ ammonium bicarbonate and $6 M$ urea and subjected to proteolytic digestion as detailed below to enhance the chances of their detection by mass spectrometric analysis.

\section{Shotgun LC-MS/MS}

Acetone Precipitation. Sample supernatants containing $250 \mu \mathrm{g}$ of protein were mixed with chilled acetone and incubated overnight at $-20^{\circ} \mathrm{C}$. The protein precipitate recovered by centrifugation at $16,000 \times g$ for $15 \mathrm{~min}$ was resuspended in $100 \mathrm{~m} M$ ammonium bicarbonate and $6 M$ urea for subsequent multi-enzyme proteolytic digestion.

Proteolytic Enzyme Digestion. To improve the digestion efficiency, a multi-enzyme digestion protocol using trypsin and endoproteinase GluC (Roche Diagnostics Deutschland GmbH, Mannheim, Germany) was used. The proteins were reduced with $10 \mathrm{~m} M$ dithiothreitol and alkylated with $40 \mathrm{~m} M$ iodoacetamide, which was followed by in-solution digestion with GluC for $6 \mathrm{~h}$ at $25^{\circ} \mathrm{C}$ followed by sequencing-grade trypsin for $16 \mathrm{~h}$ at $37^{\circ} \mathrm{C}$ (Nandakumar et al., 2011). The peptides were vacuum dried, concentrated, and stored at $-80^{\circ} \mathrm{C}$ until further analysis.

\section{NanoLC-MS/MS}

The tryptic peptide samples were subjected to nanoLC-MS/MS analysis using an LTQ-Velos Pro Dual-Pressure Linear Ion Trap mass spectrometer integrated with ETD (Thermo Scientific, San Jose, CA) and coupled to an UltiMate 3000 RSLCnano system (Dionex Corp., Sunnyvale, CA). The LC-MS/MS workflow included an online sample preconcentration and desalting step, using a monolithic $\mathrm{C}_{18}$ trap column (Pep Map, $300 \mu \mathrm{m}$ i.d. $\times 5 \mathrm{~mm}$ length, $100 \AA$ pore size, $5-\mu \mathrm{m}$ particle size; Dionex Corp.). The sample was loaded on to the monolithic trap column at a flow rate of 40 $\mu \mathrm{L} / \mathrm{min}$. The desalted peptides were then eluted and separated on a $\mathrm{C}_{18}$ Pep Map column $(75 \mu \mathrm{m}$ i.d. $\times 15$ cm length, $3-\mu \mathrm{m}$ particle size, $100 \AA$ pore size; New Objective Inc., Woburn, MA) by applying an acetonitrile gradient (acetonitrile plus $0.1 \%$ formic acid; $60 \mathrm{~min}$ ) at a flow rate of $200 \mathrm{~nL} / \mathrm{min}$ and were introduced into the mass spectrometer using the nanospray source. All MS/ MS methods for the LTQ Velos Pro mass spectrometer with ETD were set in the data-dependent acquisition mode. The LTQ Velos Pro mass spectrometer was operated with the following parameters: nanospray voltage $=2.0 \mathrm{kV}$, heated capillary temperature $=250^{\circ} \mathrm{C}$, and full-scan $m / z$ range $=400$ to 2,000 . The data acquisition was done with alternating collision-induced dissociation (CID), high-energy collision dissociation (HCD), and ETD; 6 MS/MS spectra were obtained for every full scan and 5 microscans were averaged for full scans and MS/MS scans. The normalized collision energy was set to $35 \%$ for CID, with an isolation width of 3.0 and activation time of $10 \mathrm{~ms}$. Supplemental activation was 
enabled for ETD; the activation time was set to 100 $\mathrm{ms}$ and isolation width of 3.0. The signal threshold for triggering an MS/MS event was set to 500 counts.

\section{Data Analysis and Protein Bioinformatics}

A comprehensive custom database was constructed using the protein sequences $(12,667$ entries) from $L$. acidophilus strains available in the National Center for Biotechnology Information (NCBI) database (www. ncbi.nlm.nih.gov/). This was appended with the sequences for bacteriocins (total $=177 ; 39$ from Lactobacillus spp.) compiled in the BACTIBASE database (version 2; http://bactibase.pfba-lab-tun.org). The latter was done to enhance the chances of identifying bacteriocinogenic peptides from L. acidophilus with sequence similarities to peptides belonging to bacteriocins from other Lactobacillus spp. as well as other bacteria.

The acquired MS/MS spectra were searched against this custom database with Proteome Discoverer (version 1.3; Thermo Scientific), using 3 different database search engines in a data-dependent decision tree logic: Mascot (Matrix Science Ltd., London, UK) and SEQUEST (Thermo Scientific) for CID, HCD, and ETD spectra, and Z Core (Thermo Scientific) to search ETD spectra exclusively. The search criteria included enzyme: trypsin and GluC or none, missed cleavages: 2, mass: monoisotopic, fixed modification: carbamidomethyl (C), variable modification: oxidation (M), peptide tolerance: 1.5 Da, and MS/MS fragment ion tolerance: 1 Da. Scaffold software (version 4.0; Proteome Software Inc., Portland, OR) was used to validate the peptide assignments and proteins identified by all 3 search algorithms. Peptide identifications were accepted if they could be established at greater than 90\% probability. The criteria for protein identification included the detection of at least 1 unique peptide per protein and a protein probability score of $\geq 90 \%$. Proteins that contained similar peptides and could not be differentiated based on MS/MS analysis alone were grouped to satisfy the principles of parsimony. The data sets generated from shotgun LC-MS/MS analysis of the proteolytic digests of proteins $\geq 3 \mathrm{kDa}$ (workflow 1 ) and the acetone-precipitated protein samples (workflow 2) were merged and a complied list was generated. Relative quantitation of proteins was done by the use of the label-free method of spectral counting (Liu et al., 2004) using normalized spectral counts for each protein.

\section{RESULTS}

The direct LC-MS/MS analysis of the fraction containing predominantly proteins/peptides $\leq 3 \mathrm{kDa}$ resulted in the identification of peptides belonging to
37 bacteriocins or related proteins (Table 1). Among them, 23 were high-confidence proteins identified with more than 2 unique peptides and 7 with more than 4 exclusive peptides. These bacteriocinogenic peptides were not identified in the control medium. Peptides from lactacin $\mathrm{F}$, helveticin $\mathrm{J}$, lysin, avicin $\mathrm{A}$, acidocin $\mathrm{M}$, curvaticin FS47, and carocin D were predominant in the tested samples. Peptides assigned to bacteriocins such as bavaricin (MN, A), lactococcin (A, G), and propionicin (SM1, F) were also detected in these preparations, albeit in lower abundance. Peptides belonging to many types of colicins were also identified. In the commercial preparation (NATSCO), peptides from bacteriocins such as brevicin 27 , lysin, microcin 24 , and carocin D were identified in significant abundance.

The freeze-drying process was observed to exert a significant effect on both the bacteriocin protein profile and their individual abundance. A total of 22 bacteriocins were identified in the freeze-dried preparation compared with 27 in liquid culture media, among which many were detected at lower levels in dried media such as lactacin F (4-fold). Altogether, the drying process led to a lower abundance of 17 bacteriocins, of which 12 were not detected after drying. Lysin and curvaticin were among the major bacteriocins affected by the drying step, with the abundance being reduced by 1.9-fold and 5.5-fold, respectively. However, the drying process also resulted in the concentration of 12 antimicrobial proteins; 7 of them, such as avicin A (14-fold), were below the detection limit in the liquid culture media.

The diversity and abundance of bacteriocins identified varied significantly between freeze-dried preparations from Natren Inc. (N3) and the commercial source (NATSCO) with varying culture media composition. Twenty-two bacteriocin proteins were differentially expressed in Natren Inc. samples compared with 18 in the NATSCO sample. The garbanzo beans-based media used by Natren Inc. resulted in the identification of peptides from 10 bacteriocins, such as helveticin $\mathrm{J}$ and avicin $\mathrm{A}$, which were not detected in the commercial preparation. Similarly, peptides from 6 bacteriocins were identified only in the commercial sample, among which brevicin 27 and acidocin M were prominent.

Peptide sequences assigned to the identified bacteriocins and fragmentation modes are given in Supplementary Table S1 (http://dx.doi.org/10.3168/jds.2013$7470)$. Several of the bacteriocins were assigned with more than 2 unique peptides, such as carocin D and lysin with 13 exclusive peptides, resulting in higher confidence in their identification. In addition, many of the peptides were identified by sequence consensus drawn from MS/MS spectra obtained from more than 1 fragmentation technique, enabling better assignment 
Table 1. Bacteriocins and related proteins identified by direct liquid chromatography-tandem mass spectrometry (LC-MS/MS) ${ }^{1}$

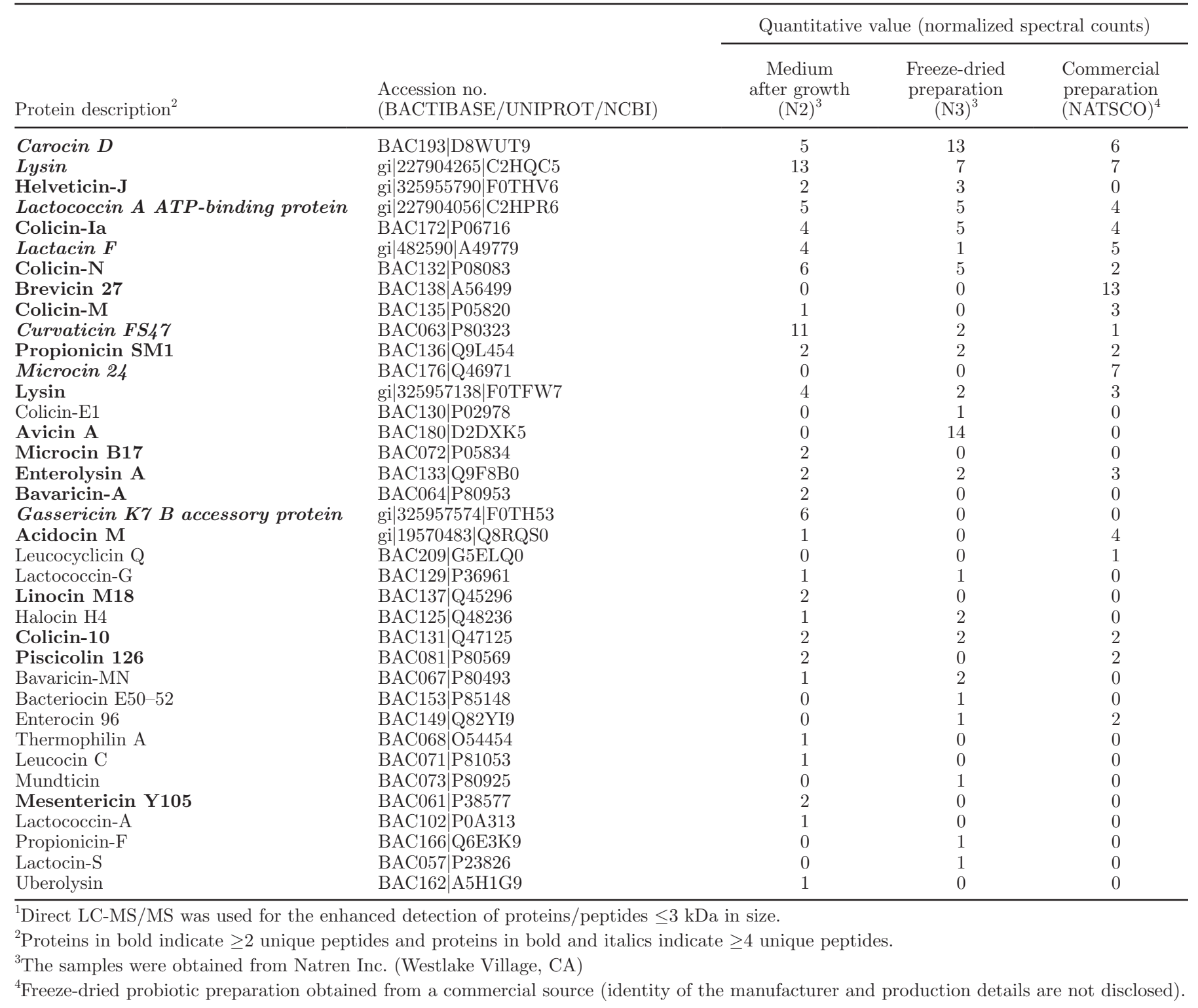

of fragment ions, leading to higher peptide probability scores and better sequence coverage.

Figure 1 shows the CID MS/MS spectra of a peptide with a precursor $m / z$ of $646.27 \mathrm{Da}$ (protonated mass $\mathrm{MH}^{+}=1,936.82 \mathrm{Da}$, charge $\left.\mathrm{Z}=+3\right)$ and retention time of $28.56 \mathrm{~min}$. The inferred peptide sequence QTNVGGAVGXAMIGATVGGTI was assigned to the bacteriocin lactacin $\mathrm{F}$, representing $84 \%$ protein sequence coverage. Lactacin $\mathrm{F}$ was identified in all 3 tested preparations from 9 peptide spectra obtained by a combination of CID, ETD, and HCD fragmentation, leading to the identification of 8 unique peptides belonging to this bacteriocin. Similarly, Figure 2 illustrates the ETD MS/MS spectra of a triply charged precursor ion with an $m / z$ of $518.41 \mathrm{Da}\left(\mathrm{MH}^{+}=1,553.24 \mathrm{Da}\right)$, with a retention time of $30.44 \mathrm{~min}$. The deduced peptide sequence MLIATMENDGTGHF was assigned to the bacteriocin helveticin $\mathrm{J}$ from $L$. acidophilus, which was identified with 4 unique peptides and 5 peptide spectra derived from both HCD and ETD peptide spectral matches.

Shotgun LC-MS/MS analysis of the GluC-tryptic peptides obtained from a multi-enzyme digest of the samples resulted in the identification of 1,287 proteins and among them only 15 were reported to be bacteriocins or related proteins (Table 2). Moreover, all the bacteriocins were detected at a very low abundance level compared with direct LC-MS/MS. However, pep- 


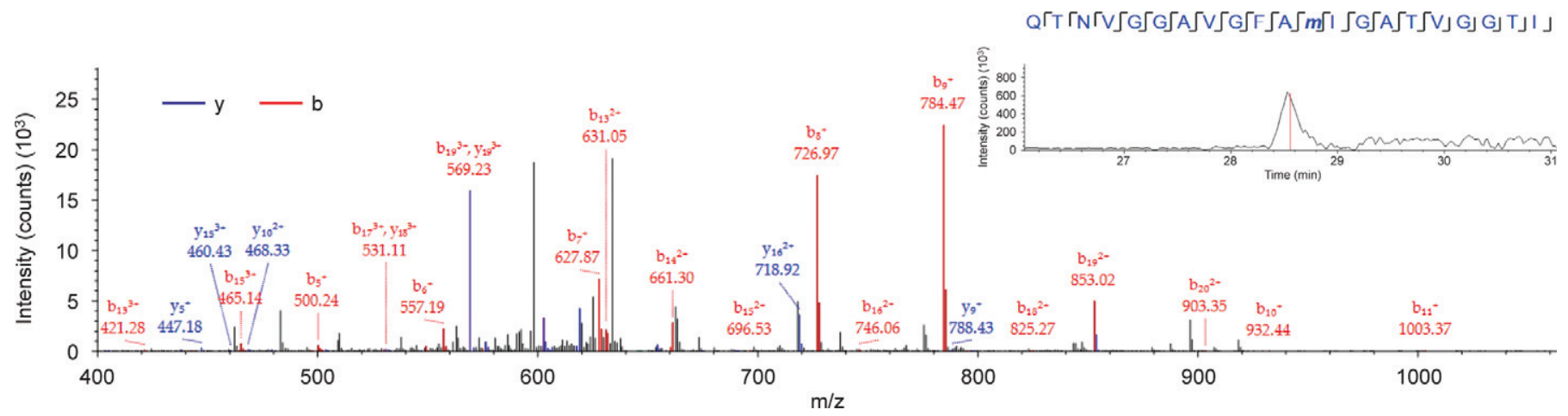

Figure 1. Collision-induced dissociation (CID)-based tandem mass spectrometry (MS/MS) spectra of the peptide QTNVGGAVGXAMIGATVGGTI assigned to lactacin F (precursor monoisotopic $m / z: 646.27 \mathrm{Da}$; protonated mass $\mathrm{MH}^{+}=1,936.82 \mathrm{Da}$; charge Z $=+3)$. Matched b- and y-ions are indicated in the spectra. An extracted ion chromatogram (XIC) of the precursor $(\mathrm{m} / z: 646.27$; retention time: $28.56 \mathrm{~min}$ ) is shown in the inset. The vertical line indicates the retention time of the precursor ion. Color version available in the online PDF.

tides from bacteriocins such as dysgalacticin, pyocin, and thermophilin 1277 were identified only after multienzyme digestion. Bacteriocin helveticin J, which was not identified by direct LC-MS/MS in the commercial preparation, was detected in high abundance after shotgun analysis.

\section{DISCUSSION}

Bacteriocins are a very heterogeneous group and can be either small peptides consisting of 19 to 37 amino acids with molecular weights $<5$ to $10 \mathrm{kDa}$ or larger peptides with molecular weights up to $90 \mathrm{kDa}$. Bacteriocin production has been suggested as a key probiotic trait and L. acidophilus is known to secrete a diverse array of bacteriocins, such as lactacin B, F, and acidocin (Chumchalová et al., 2004; de Souza et al., 2005). Bacteriocins of L. acidophilus, from their production to application, are summarized in Ahmed et al. (2010).

Application of mass spectrometry in bacteriocin research so far has been limited mostly as a supplementary technique for the mass determination of purified antibacterial peptides, and MALDI-TOF-MS seems to be the preferred method of choice. Zendo et al. (2008) applied ESI-LC-MS for the rapid identification of nisin and lactacin from LAB and, recently, Perez et al. (2012) proposed ESI-LC-MS for the quantification of enterocins from Enterococcus faecium. All these analytical strategies are limited in scope, require prior knowledge of proteins/peptides being identified, and do not provide any sequence information on the novel bacteriocins. The heterogeneity of bacteriocins demands a more advanced nanoLC-MS/MS analysis, characterized by its high sensitivity, specificity, reproducibility, and wide dynamic range (Gaspari and Cuda, 2011) and this technique is especially suited for the identification of small-molecular-weight, low-abundant bacteriocinogenic peptides. A bottom-up, shotgun proteomic approach using an analytical platform integrating nanoLC-MS/ MS analysis with advanced fragmentation techniques coupled with extensive bioinformatic data mining has not been exploited in bacteriocin research until now.

This study was done with the objective of identifying the bacteriocins present in the culture preparations of

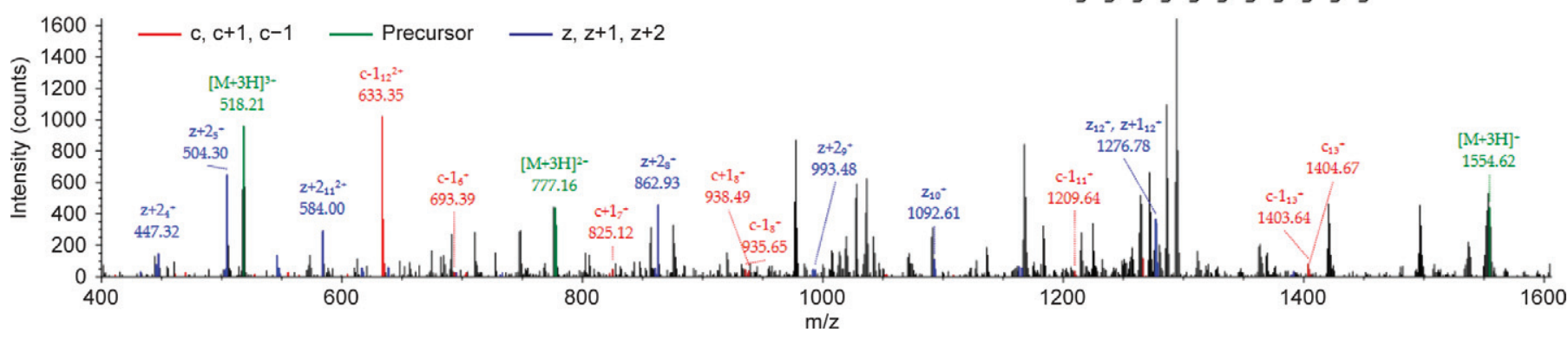

Figure 2. Electron-transfer dissociation (ETD)-based tandem mass spectrometry (MS/MS) spectra of the peptide MLIATMENDGTGHF assigned to the bacteriocin helveticin $\mathrm{J}$ (precursor monoisotopic $m / z: 518.41 \mathrm{Da}$; protonated mass $\mathrm{MH}^{+}=1,553.24 \mathrm{Da}$; charge $\mathrm{Z}=+3$; retention time: $30.44 \mathrm{~min}$ ). Matched z1, z2, and c-fragment ions are indicated in the spectra. Color version available in the online PDF. 
Table 2. Bacteriocins and related proteins identified by shotgun liquid chromatography-tandem mass spectrometry (LC-MS/MS) ${ }^{1}$

\begin{tabular}{|c|c|c|c|c|}
\hline Protein description & $\begin{array}{l}\text { Accession no. } \\
\text { (BACTIBASE/UNIPROT/ } \\
\text { NCBI) }\end{array}$ & \multicolumn{3}{|c|}{ Quantitative value (normalized spectral counts) } \\
\hline Propionicin SM1 & BAC136|Q9L454 & 3 & 4 & 0 \\
\hline Dysgalacticin & BAC186; Q14DY1 & 1 & 0 & 0 \\
\hline Lactacin F & gi $|482590| \mathrm{A} 49779$ & 0 & 1 & 0 \\
\hline Response regulator bacteriocin production & gi|58336928|Q5FLE2 & 1 & 2 & 0 \\
\hline Colicin-10 & BAC131|Q47125 & 0 & 0 & 2 \\
\hline Colicin-N & BAC132|P08083 & 1 & 0 & 0 \\
\hline Linocin M18 & BAC137|Q45296 & 1 & 1 & 0 \\
\hline Gassericin K7 B accessory protein & gi|325957574|F0TH53 & 1 & 1 & 0 \\
\hline Thermophilin 1277 & BAC155|A7M695 & 0 & 1 & 0 \\
\hline Enterolysin A & gi|227904125|Q5FJT1 & 1 & 2 & 0 \\
\hline
\end{tabular}

L. acidophilus to assess their probiotic potential. The culture media used in this study promoted the secretion of many peptides belonging to bacteriocins, such as lactacin $\mathrm{F}$, helveticin $\mathrm{J}$, lysin, avicin $\mathrm{A}$, acidocin $\mathrm{M}$, curvaticin FS47, and carocin D, to significant levels. The major bacteriocins of LAB and their main characteristics are listed by Parada et al. (2007). Lactacin F is a heat-stable, 2.5-kDa bacteriocin reported to be active against Enterococcus faecalis and other lactobacilli such as Lactobacillus fermentum, Lactobacillus delbrueckii, and Lactobacillus helveticus (Muriana and Klaenhammer, 1991). Another type, lactacin B, produced by $L$. acidophilus, was not detected in the present study. Acidocin from $L$. acidophilus is known to have restricted activity against gram-positive bacteria and one $6.6-\mathrm{kDa}$ acidocin was reported to be extremely heat stable, with activity over a wide $\mathrm{pH}$ range (Deraz et al., 2005). In our samples, a very low abundant acidocin $\mathrm{M}$ was identified in the culture medium before freeze drying. The instrumentation platform with a high mass accuracy and resolution used here was capable of distinguishing between different types of bacteriocins with close molecular masses.

We identified many peptides in our samples with sequence similarities to peptides assigned to bacteriocins produced by species other than L. acidophilus. It is plausible that these peptides were originated from homologous proteins; however, a BLAST search (http:// blast.ncbi.nlm.nih.gov/Blast.cgi) of the identified peptide sequences against NCBInr protein databases did not result in any other significant matches with high expect ratio, except with those bacteriocins to which they were assigned. Moreover, most of these identified bacteriocins had more than 2 unique peptides assigned to them, especially those with sequence similarities to carocin D, curvaticin FS47 and microcin 24, with 13, 7, and 4 exclusive peptides, leading to higher confidence in their identification. Colicin production proteins are identified in several Lactobacillus genomes and a putative uncharacterized protein (Q5FI42; UniProtKB; http://www.uniprot.org/help/uniprotkb) is reported in L. acidophilus, with signature matches to the colicin $\mathrm{V}$ production family. Håvarstein et al. (1994) showed through mass spectrometry analysis that the leader peptide of colicin $\mathrm{V}$ shares consensus sequences with leader peptides that are common among bacteriocins produced by many LAB. The LTQ Velos Pro mass spectrometer used in the present study with sensitivity at the femtomole level also might have contributed significantly to the identification of these low-abundant antibacterial peptides. However, utilization of complementary techniques such as Western blots or bioassays is warranted to validate the presence of these bacteriocinogenic peptides in the samples.

In the food and pharmaceutical industries, drying of bacterial cells by different techniques such as freeze drying, spray drying, or low-temperature vacuum drying is often used for product conservation (Wang et al., 2004; Wong et al., 2007). Freeze drying was suggested as a feasible approach for the preparation of powdered bacteriocins for prospective food applications (DimitrievaMoats and Ünlü, 2012). The process of freeze drying under vacuum applied here for the product formulation was observed to significantly influence both the 
diversity and abundance of the bacteriocins. Recently, Jahn et al. (2013) compared the effects of preservation methods such as freeze drying and vacuum drying on the proteome profile of Pseudomonas putida analyzed by LC-MS/MS. It was reported that although the overall proteome profile of freeze-dried and vacuum-dried samples were significantly similar to the fresh samples, the quantity of proteins belonging to certain functional categories were more prone to alteration than others. The effects of storage temperature and duration on the proteomic profile has been studied more extensively in human serum and plasma samples, highlighting a differential effect between proteins and sample types (Pieragostino et al., 2010; Pasella et al., 2013). Freeze drying has been suggested to improve the storage stability of proteins; however, many proteins are susceptible to cold denaturation or denaturation at protein-air and protein-ice interfaces (Tang and Pikal, 2004). In addition, this process generates a variety of freezing and drying stresses, such as changes in ionic strength, water content, protein concentration, and localized $\mathrm{pH}$ shifts, leading to denaturation or aggregation of sensitive proteins (Wang, 2000). However, the integrity of proteins within whole cells during cold storage is not well known. The alterations in bacteriocin expression profile and abundance observed between fresh and freeze-dried samples could be attributed to the variations in the tolerance of different proteins to withstand the dynamic stresses imposed by freeze drying. Further bioassays are warranted to assess the extent to which processing parameters affect the bioactive potential of these bacteriocins.

Two freeze-dried probiotic preparations (N3 and NATSCO) with different production pipeline but no strain variability were compared to evaluate the role of growth factors, freeze-drying parameters, and formulation composition on the bacteriocin profile. Several studies have documented the effect of nutritional factors and culture conditions, especially $\mathrm{pH}$, temperature, carbon and nitrogen sources, and $\mathrm{NaCl}$ concentration on bacteriocin production (Guerra et al., 2001; Todorov and Dicks, 2005; Mahrous et al., 2013). The growth medium has been cited as a critical parameter in the freeze-drying tolerance of microorganisms, as it may influence accumulation of compatible solutes, production of exopolysaccharides, and altered FA profile of the membrane (Carvalho et al., 2004). Freeze-drying tolerance also depends on the freeze-drying medium, freezing rate, freeze-drying parameters, rehydration conditions, and initial cell concentration (Shafiei et al., 2013). The significantly different bacteriocin protein profiles and their differential expression detected between these 2 samples highlight the effects of bacterial growth and process parameters on the final nutritional value of probiotic products. From an analytical point of view, the proposed approach integrating size-exclusion prefractionation and LC-MS/MS workflow is proven to be effective for both qualitative and quantitative identification of low-abundant bacteriocins from samples of varying complexity.

Electron-transfer dissociation is a recently introduced fragmentation technique in MS/MS that produces c- and z-type fragment ions and is particularly useful for peptides with charge states $>2$ (Sobott et al., 2009; Frese et al., 2011). Complementary to CID fragmentation, ETD has several advantages, particularly for the analysis of large, high-charge peptides or undigested proteins and labile modifications (Quan and Liu, 2013). Acquisition protocol applied in the current study involving complementary, alternative peptide fragmentation methods such as CID, HCD, and ETD on the same precursor ion were shown to substantially increase the confidence and extent of fragment ion matches and improve identification of low-abundant peptides. Bioinformatic approaches, including custom databases, multiple search engines, and validation software with different scoring algorithms to maximize and cross-validate protein identification, also might have contributed to expanding the breadth of the bacteriocin protein profile in the current study.

The shotgun LC-MS/MS workflow where proteins were subjected to an enrichment step based on acetone precipitation and multi-enzyme digestion before mass spectrometry analysis did not result in the identification of as many bacteriocins as expected. This could be attributed to the increased sample complexity and peptide dynamic range as a consequence of the multienzyme digestion of all the proteins, which might have prevented the detection of low-abundant bacteriocin peptides. No attempt was made to extract the bacteriocins possibly adsorbed onto the cell surface, as the main objective of this study was to identify the bacteriocins present extracellularly in the culture media. Moreover, these tested preparations had a final $\mathrm{pH}$ of 4.63 , well below $\mathrm{pH}$ 6.0, where the adsorption of bacteriocins onto producer cells was reported to be maximum (Yang et al., 1992).

Emergence of bacteriocin-resistant or bacteriocintolerant bacterial strains is a serious concern, impeding the large-scale commercial application of bacteriocins (Guinane et al., 2006). Different strategies have been suggested to circumvent this concern, including a combination of bacteriocins that differ in kinetics of inhibition and cross-resistance of survivors (Vignolo et al., 2000). Another better, alternative strategy is the utilization of strains that can produce multiple bacteriocins with different target spectra, host-cell interaction and killing mechanisms, and inhibition kinetics that are 
complementary and cumulative. From that point of view, the strain used in this study with its capability to produce a diverse array of bacteriocinogenic peptides to significant abundance levels would be a suitable candidate for further validation assays to assess its probiotic benefits.

The analytical approach described here, incorporating size-based fractionation, nanoLC-MS/MS analysis, mass spectrometric data acquisition using multiple fragmentation techniques, bioinformatic approaches such as deriving peptide consensus from different activation modalities, and data mining with multiple search engines for the global profiling of bacteriocins, is novel and comprehensive. The strategies described here resulted in the identification of a wide array of bacteriocin peptides in all tested probiotic preparations of $L$. acidophilus, which could enhance its potential probiotic benefits.

\section{ACKNOWLEDGMENTS}

The mass spectrometry analysis was done at the Proteomics and Metabolomics Core Facility (Redox Biology Center, University of Nebraska-Lincoln, Lincoln), supported by the National Institutes of Health (NIH, Bethesda, MD; P30GM103335).

\section{REFERENCES}

Abee, T. 1995. Pore-forming bacteriocins of gram-positive bacteria and self-protection mechanisms of producer organisms. FEMS Microbiol. Lett. 129:1-10.

Aebersold, R., and M. Mann. 2003. Mass spectrometry-based proteomics. Nature 422:198-207.

Ahmed, Z., Y. Wang, Q. Cheng, and M. Imran. 2010. Lactobacillus acidophilus bacteriocin, from production to their application: An overview. Afr. J. Biotechnol. 9:2843-2850.

Carvalho, A. S., J. Silva, P. Ho, P. Teixeira, F. X. Malcata, and P. Gibbs. 2004. Relevant factors for the preparation of freeze-dried lactic acid bacteria. Int. Dairy J. 14:835-847.

Chen, H., and D. G. Hoover. 2003. Bacteriocins and their food applications. Compr. Rev. Food Sci. Food Saf. 2:82-100.

Chumchalová, J., J. Stiles, J. Josephsen, and M. Plocková. 2004. Characterization and purification of acidocin $\mathrm{CH} 5$, a bacteriocin produced by Lactobacillus acidophilus CH5. J. Appl. Microbiol. 96:1082-1089.

Cintas, L. M., M. P. Casaus, C. Herranz, I. F. Nes, and P. E. Hernández. 2001. Review: Bacteriocins of lactic acid bacteria. Food Sci. Technol. Int. 7:281-305.

Corr, S. C., C. Hill, and C. G. M. Gahan. 2009. Understanding the mechanisms by which probiotics inhibit gastrointestinal pathogens. Adv. Food Nutr. Res. 56:1-15.

Cotter, P. D., C. Hill, and R. P. Ross. 2005. Bacteriocins: Developing innate immunity for food. Nat. Rev. Microbiol. 3:777-788.

de Souza, E. L., C. A. da Silva, and C. P. de Sousa. 2005. Bacteriocins: Molecules of fundamental impact on the microbial ecology and potential food preservatives. Braz. Arch. Biol. Technol. 48:559-566.

Deraz, S. F., E. N. Karlsson, M. Hedström, M. M. Andersson, and B. Mattiasson. 2005. Purification and characterization of acidocin D20079, a bacteriocin produced by Lactobacillus acidophilus DSM 20079. J. Biotechnol. 117:343-354.
Dimitrieva-Moats, G. Y., and G. Ünlü. 2012. Development of freezedried bacteriocin-containing preparations from lactic acid bacteria to inhibit Listeria monocytogenes and Staphylococcus aureus. Probiotics and Antimicrobial Proteins 4:27-38.

Dobson, A., P. D. Cotter, R. P. Ross, and C. Hill. 2012. Bacteriocin production: A probiotic trait? Appl. Environ. Microbiol. 78:1-6.

Frese, C. K., A. F. M. Altelaar, M. L. Hennrich, D. Nolting, M. Zeller, J. Griep-Raming, A. J. R. Heck, and S. Mohammed. 2011. Improved peptide identification by targeted fragmentation using CID, HCD and ETD on an LTQ-Orbitrap Velos. J. Proteome Res. 10:2377-2388.

Gaspari, M., and G. Cuda. 2011. Nano LC-MS/MS: A robust setup for proteomic analysis. Methods Mol. Biol. 790:115-126.

Gillor, O., A. Etzion, and M. A. Riley. 2008. The dual role of bacteriocins as anti- and probiotics. Appl. Microbiol. Biotechnol. 81:591-606.

Good, D. M., M. Wirtala, G. C. McAlister, and J. J. Coon. 2007. Performance characteristics of electron transfer dissociation mass spectrometry. Mol. Cell. Proteomics 6:1942-1951.

Guerra, N. P., M. L. Rua, and L. Pastrana. 2001. Nutritional factors affecting the production of two bacteriocins from lactic acid bacteria on whey. Int. J. Food Microbiol. 70:267-281.

Guinane, C. M., P. D. Cotter, C. Hill, and R. P. Ross. 2006. Spontaneous resistance in Lactococcus lactis IL1403 to the lantibiotic lacticin 3147. FEMS Microbiol. Lett. 260:77-83.

Håvarstein, L. S., H. Holo, and I. F. Nes. 1994. The leader peptide of colicin $\mathrm{V}$ shares consensus sequences with leader peptides that are common among peptide bacteriocins produced by gram-positive bacteria. Microbiology 140:2382-2389.

Hindré, T., S. Didelot, J.-P. Le Pennec, D. Haras, A. Dufour, and K. Vallée-Réhel. 2003. Bacteriocin detection from whole bacteria by matrix-assisted laser desorption ionization-time of flight mass spectrometry. Appl. Environ. Microbiol. 69:1051-1058.

Jack, R. W., J. R. Tagg, and B. Ray. 1995. Bacteriocins of grampositive bacteria. Microbiol. Rev. 59:171-200.

Jahn, M., J. Seifert, T. Hübschmann, M. Bergen, H. Harms, and S. Müller. 2013. Comparison of preservation methods for bacterial cells in cytomics and proteomics. J. Integrated OMICS 3:25-33.

Klaenhammer, T. R. 1993. Genetics of bacteriocins produced by lactic acid bacteria. FEMS Microbiol. Rev. 12:39-85.

Liu, H., R. G. Sadygov, and J. R. Yates III. 2004. A model for random sampling and estimation of relative protein abundance in shotgun proteomics. Anal. Chem. 76:4193-4201.

Mahrous, H., A. Mohamed, M. Abd El-Mongy, A. I. El-Batal, and H. A. Hamza. 2013. Study bacteriocin production and optimization using new isolates of Lactobacillus spp. isolated from some dairy products under different culture conditions. Food Nutr. Sci. 4:342-356.

Malini, M., and S. Janakiraman. 2012. Detection of heat stable bacteriocin from Lactobacillus acidophilus NCIM5426 by liquid chromatography/mass spectrometry. Indian J. Sci. Technol. 5:2325-2332.

MarketsandMarkets. 2009. Probiotic market-advanced technologies and global market (2009-2014). Report Code: FB 1046. MarketsandMarkets, Dallas, TX.

Mugochi, T., M. P. Nandakumar, R. Zvauya, and B. Mattiasson. 2001. Bioassay for the rapid detection of bacteriocins in fermentation broth. Biotechnol. Lett. 23:1243-1247.

Muriana, P. M., and T. R. Klaenhammer. 1991. Purification and partial characterization of lactacin F, a bacteriocin produced by Lactobacillus acidophilus. Appl. Environ. Microbiol. 57:114-121.

Nandakumar, R., C. Espirito Santo, N. Madayiputhiya, and G. Grass 2011. Quantitative proteomic profiling of the Escherichia coli response to metallic copper surfaces. Biometals 24:429-444.

Nandakumar, R., M. P. Nandakumar, and B. Mattiasson. 2000. Quantification of nisin in flow-injection immunoassay systems. Biosens. Bioelectron. 15:241-247.

Nesvizhskii, A. I. 2007. Protein identification by tandem mass spectrometry and sequence database searching. Methods Mol. Biol. $367: 87-119$. 
Parada, J. L., C. R. Caron, A. B. P. Medeiros, and C. R. Soccol. 2007. Bacteriocins from lactic acid bacteria: Purification, properties and use as biopreservatives. Braz. Arch. Biol. Technol. 50:521-542.

Pasella, S., A. Baralla, E. Canu, S. Pinna, J. Vaupel, M. Deiana, C. Franceschi, G. Baggio, A. Zinellu, S. Sotgia, G. Castaldo, C. Carru, and L. Deiana. 2013. Pre-analytical stability of the plasma proteomes based on the storage temperature. Proteome Sci. 11:10.

Perez, R. H., K. Himeno, N. Ishibashi, Y. Masuda, T. Zendo, K. Fujita, P. Wilaipun, V. Leelawatcharamas, J. Nakayama, and K. Sonomoto. 2012. Monitoring of the multiple bacteriocin production by Enterococcus faecium NKR-5-3 through a developed liquid chromatography and mass spectrometry-based quantification system. J. Biosci. Bioeng. 114:490-496.

Pieragostino, D., F. Petrucci, P. Del Boccio, D. Mantini, A. Lugaresi, S. Tiberio, M. Onofrj, D. Gambi, P. Sacchetta, C. Di Ilio, G. Federici, and A. Urbani. 2010. Pre-analytical factors in clinical proteomics investigations: Impact of ex vivo protein modifications for multiple sclerosis biomarker discovery. J. Proteomics 73:579-592.

Quan, L., and M. Liu. 2013. CID, ETD and HCD fragmentation to study protein post-translational modifications. Mod. Chem. Appl. $1: 1-5$.

Riley, M. A., and J. E. Wertz. 2002. Bacteriocins: Evolution, ecology, and application. Annu. Rev. Microbiol. 56:117-137.

Rose, N. L., P. Sporns, and L. M. McMullen. 1999. Detection of bacteriocins by matrix-assisted laser desorption/ionization time-of-flight mass spectrometry. Appl. Environ. Microbiol. 65:2238-2242.

Sawa, N., S. Koga, K. Okamura, N. Ishibashi, T. Zendo, and K. Sonomoto. 2013. Identification and characterization of novel multiple bacteriocins produced by Lactobacillus sakei D98. J. Appl. Microbiol. 115:61-69.

Shafiei, R., F. Delvigne, and P. Thonart. 2013. Flow-cytometric assessment of damages to Acetobacter senegalensis during freeze-drying process and storage. Acetic Acid Bacteria 2:62-70.

Sobott, F., S. J. Watt, J. Smith, M. J. Edelmann, H. B. Kramer, and B. M. Kessler. 2009. Comparison of CID versus ETD based MS/
MS fragmentation for the analysis of protein ubiquitination. J Am. Soc. Mass Spectrom. 20:1652-1659.

Tang, X., and M. J. Pikal. 2004. Design of freeze-drying processes for pharmaceuticals: Practical advice. Pharm. Res. 21:191-200.

Todorov, S. D., and L. M. T. Dicks. 2005. Effect of growth medium on bacteriocin production by Lactobacillus plantarum ST194BZ, a strain isolated from Boza. Food Technol. Biotechnol. 43:165-173.

Urbizu, L., M. Sparo, and S. S. Bruni. 2013. Bacterial antagonist mediated protein molecules. Clin. Exp. Pharmacol. 3:123-133.

Vignolo, G., J. Palacios, M. E. Farías, F. Sesma, U. Schillinger, W Holzapfel, and G. Oliver. 2000. Combined effect of bacteriocins on the survival of various Listeria species in broth and meat system. Curr. Microbiol. 41:410-416.

Wang, W. 2000. Lyophilization and development of solid protein pharmaceuticals. Int. J. Pharm. 203:1-60.

Wang, Y. C., R. C. Yu, and C. C. Chou. 2004. Viability of lactic acid bacteria and bifidobacteria in fermented soymilk after drying, subsequent rehydration and storage. Int. J. Food Microbiol. 93:209-217.

Wong, Y.-L., S. Sampson, W. A. Germishuizen, S. Goonesekera, G. Caponetti, J. Sadoff, B. R. Bloom, and D. Edwards. 2007. Drying a tuberculosis vaccine without freezing. Proc. Natl. Acad. Sci. USA 104:2591-2595.

Yang, R., M. C. Johnson, and B. Ray. 1992. Novel method to extract large amounts of bacteriocins from lactic acid bacteria. Appl. Environ. Microbiol. 58:3355-3359.

Zamfir, M., R. Callewaert, P. C. Cornea, L. Savu, I. Vatafu, and L. De Vuyst. 1999. Purification and characterization of a bacteriocin produced by Lactobacillus acidophilus IBB 801. J. Appl. Microbiol. 87:923-931.

Zendo, T., J. Nakayama, K. Fujita, and K. Sonomoto. 2008. Bacteriocin detection by liquid chromatography mass spectrometry for rapid identification. J. Appl. Microbiol. 104:499-507. 